\title{
CHAOTIC BEHAVIOR IN A TWO-DIMENSIONAL BUSINESS CYCLE MODEL
}

\author{
CRISTINA JANUÁRIO \\ Department of Chemistry, Mathematics Unit, Instituto Superior de Engenharia \\ de Lisboa, Rua Conselheiro Emídio Navarro, 1949-014 Lisboa, Portugal \\ E-mail: cjanuario@deq.isel.ipl.pt \\ CLARA GRÁCIO \\ Department of Mathematics, Universidade de Évora, Rua Romão Ramalho, 59, \\ 7000-585 Évora, Portugal \\ E-mail:mgracio@uevora.pt

\section{J. SOUSA RAMOS} \\ Department of Mathematics, Instituto Superior Técnico, Av. Rovisco Pais 1, \\ 1049-001 Lisboa, Portugal \\ E-mail:sramos@math.ist.utl.pt

\begin{abstract}
We consider a discrete-time economic model which is a particular case of the Kaldor-type business cycle model and it is described by a two-dimensional dynamical system. Under certain conditions the map can be reduced to a skew map whose components, the base and the fiber map, both have entropy. Our proposal is to study and measure the complexity of the system using symbolic dynamics techniques and the topological entropy.
\end{abstract}

\section{Introduction}

The manifestation of nonlinear effects can be easily detected in almost all real world systems. In the particular case of economics, many of its important fluctuations arise out of nonlinear dynamic phenomena. The most interesting theories of business cycles in the Keynesian vein are the ones that utilizes non-linear functions. In this work we present a variation of the model considered by Bischi et al. ${ }^{2}$ and Dieci et al. ${ }^{3}$. It departs from a discrete time-version of the Kaldor model described by a two-dimensional system. We intend to introduce nonlinearity in a variable of the model in order to study the effect it produces. 
Firstly, consider the Kaldor-type business cycle model

$$
\left\{\begin{array}{l}
Y_{t+1}-Y_{t}=\alpha\left(I_{t}-S_{t}\right) \\
K_{t+1}=(1-\delta) K_{t}+I_{t}
\end{array}\right.
$$

which depends on four variables, $Y, K, I$ and $S$, representing, respectively, income, capital stock, investment and savings. We have also two parameters, $\alpha$ and $\delta$, verifying the inequalities, $\alpha>0$ and $0<\delta<1$. The parameter $\alpha$ represents the speed of reaction to the excess demand and $\delta$ is the capital stock depreciation rate.

The function describing investment is assumed to take the form of an increasing arc tangent type function of income, like suggested in Bischi et al. ${ }^{2}$ and Dieci et al. ${ }^{3}$. Since Kaldor ${ }^{5}$ concluded it might be sensible to assume that the $S$ and $I$ curves are nonlinear, in general, he assumed $I=I(Y, K)$ and $S=S(Y, K)$, where investment and savings are nonlinear functions of income and capital. In order to introduce nonlinearity to the capital stock variable, instead of considering it just a linear decreasing function, we shall propose it as the sum of two functions: one linear decreasing and other a trigonometric function of the sin type. This sum, depending on the parameters, is monotone decreasing or has consecutive decreasing local maxima (or minima) when $K$ increases (see Fig.1). Therefore, let

$$
I_{t}=\sigma \mu+\gamma_{1}\left(\frac{\sigma \mu}{\delta}-K_{t}\right)+\arctan \left(Y_{t}-\mu\right)-\beta \sin \left(0.2 K_{t}-\mu\right),
$$

where the parameters $\sigma$ and $\delta$ are such that $0<\sigma, \delta<1$ and the parameters $\beta, \gamma_{1}, \mu, \sigma$ are positive $\left(\beta, \gamma_{1}, \mu, \sigma>0\right)$.
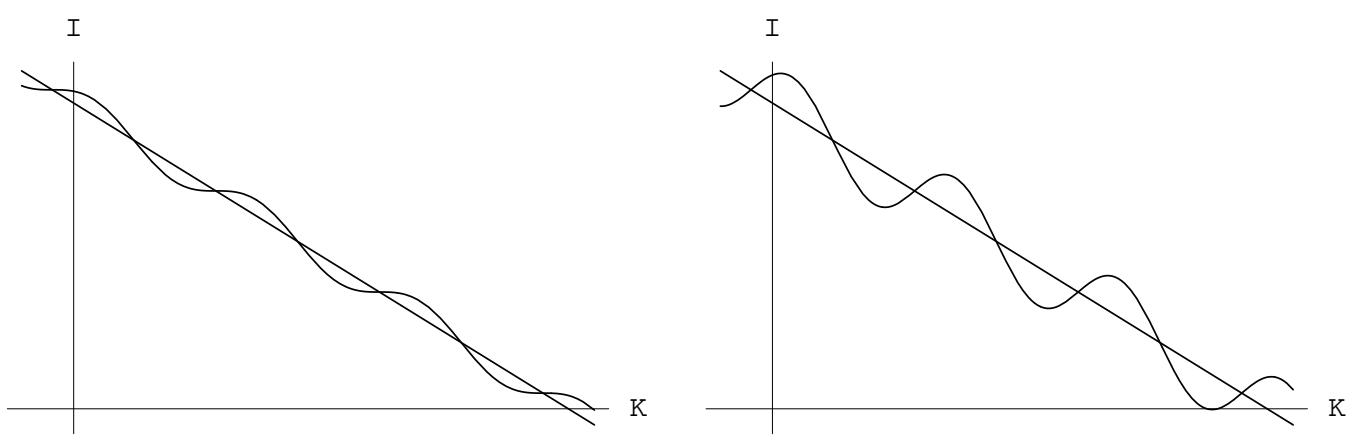

Figure 1. Effect of the nonlinearity in the capital stock variable, $K$. In plot $a$ ) $\gamma_{1}=$ $2.0, \beta=10, \mu=100$ and in plot $b) \gamma_{1}=0.8, \beta=9.5, \mu=100$. 
Concerning the savings function, we also modify the assumption of proportionality to the income assuming, as in many versions of the Kaldor business cycle models, that savings are nonlinear. We consider that it depends also on the capital stock in a decreasing way:

$$
S_{t}=\sigma Y_{t}+\gamma_{2}\left(\frac{\sigma \mu}{\delta}-K_{t}\right)-\beta \sin \left(0.2 K_{t}-\mu\right),
$$

with $\gamma_{2}$ a positive parameter.

Replacing expressions (2) and (3) in (1) and considering the particularly interesting case when $\gamma_{1}=\gamma_{2}=\gamma$ we get the following two dimensional system in income and capital stock variables:

$$
\left\{\begin{array}{l}
Y_{t+1}=Y_{t}+\alpha \sigma \mu+\alpha \arctan \left(Y_{t}-\mu\right)-\alpha \sigma Y_{t} \\
K_{t+1}=(1-\delta) K_{t}+\sigma \mu+\gamma\left(\frac{\sigma \mu}{\delta}-K_{t}\right)+\arctan \left(Y_{t}-\mu\right)-\beta \sin \left(0.2 K_{t}-\mu\right)
\end{array} .\right.
$$

This paper is organized as follows: in Section 2 we present the model and the main results concerning triangular maps and the topological entropy. In Section 3 we explore its complex dynamic behavior. We also illustrate the computation of the topological invariant in an example and finally in Section 4 we make some final considerations.

\section{The model and some considerations about triangular maps}

Let us change the notation in the model (4) to: $y:=Y$ and $k:=K$. We consider a family of two-dimensional continuously differentiable maps $F: \mathbb{R}^{2} \longrightarrow \mathbb{R}^{2}$ given by

$$
F:\left(\begin{array}{l}
y \\
k
\end{array}\right)=\left(\begin{array}{c}
y+\alpha \sigma \mu+\alpha \arctan (y-\mu)-\alpha \sigma y \\
(1-\delta) k+\sigma \mu+\gamma\left(\frac{\sigma \mu}{\delta}-k\right)+\arctan (y-\mu)-\beta \sin (0.2 k-\mu)
\end{array}\right),
$$

where $\sigma, \delta, \beta, \gamma, \mu, \sigma$ are real parameters such that $\alpha>0,0<\sigma\langle 1, \mu\rangle$ $0,0<\delta<1, \gamma>0, \beta>0$.

This dynamical system is generated by a skew map (triangular map) since it has the form $F(y, k)=(f(y), g(y, k))$, that is, the first component doesn't depend on the second variable. So, from the economic point of view, the dynamics of income is only affected by income itself. The map $f$ is called the basis map and $g$ is called the fiber map. Due to this triangular structure it is possible to apply mathematical methods to compute relevant quantities that characterize the system as chaotic or non chaotic. In Fig.2 we present the graphical representation of the map $F$ for some values of the parameters. 

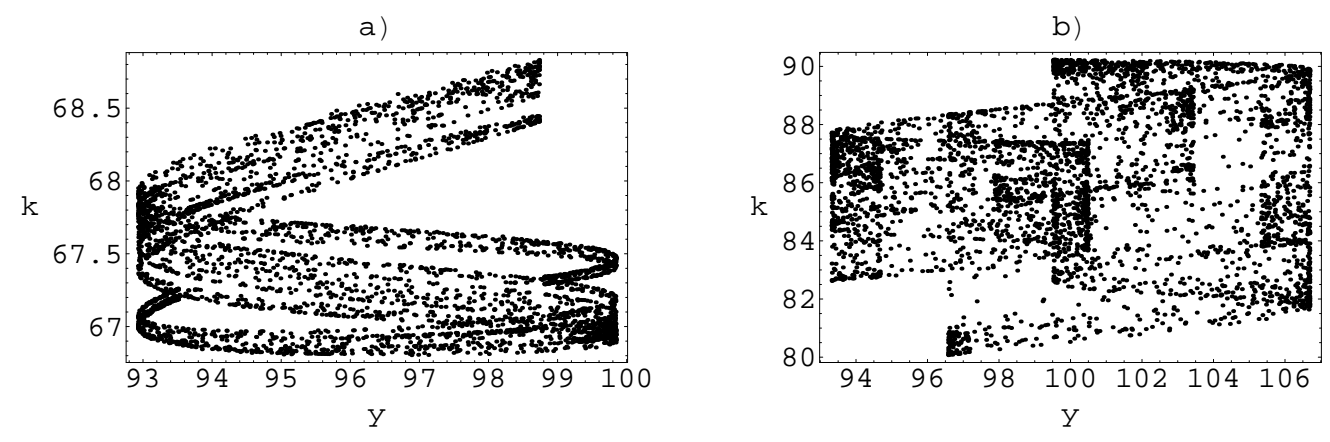

Figure 2. Graphical representation of the map $F$ for some values of the parameters. In plot a) $\alpha=10, \mu=100, \sigma=0.3, \delta=0.4, \beta=9.5, \gamma=1.0$ and in plot $b) \alpha=10$, $\mu=100, \sigma=0.32, \delta=0.4, \beta=9.5, \gamma=0.8$.

Let $P=\left\{x_{0}, x_{1}, \ldots, x_{p-1}\right\}$ be a periodic orbit of period $p$ of the map $f$ such that $f\left(x_{i}\right)=x_{i+1}$ for $i=0, \ldots, p-2$ and $f\left(x_{p-1}\right)=x_{0}$. We define the map $g_{p}: Y \longrightarrow Y$ as

$$
g_{p}(y)=g\left(x_{p-1}, g\left(x_{p-2}, \ldots g\left(x_{1}, g\left(x_{0}, y\right)\right) \ldots\right)\right) .
$$

If $Q=\left\{y_{0}, y_{1}, \ldots, y_{q-1}\right\}$ is a periodic orbit of period $q$ of the map $g_{p}$ such that $g_{p}\left(y_{i}\right)=y_{i+1}$ for $i=0, \ldots, q-2$ and $g_{p}\left(y_{p-1}\right)=y_{0}$, we can define the product $P . Q$ as the set containing the p.q pairs:

$$
\begin{array}{cccc}
\left(x_{0}, y_{0}\right) & \left(x_{1}, g\left(x_{0}, y_{0}\right)\right) & \ldots & \left(x_{p-1}, g\left(x_{p-2}, \ldots g\left(x_{1}, g\left(x_{0}, y_{0}\right)\right) \ldots\right)\right) \\
\left(x_{0}, y_{1}\right) & \left(x_{1}, g\left(x_{0}, y_{1}\right)\right) & \ldots & \left(x_{p-1}, g\left(x_{p-2}, \ldots g\left(x_{1}, g\left(x_{0}, y_{1}\right)\right) \ldots\right)\right) \\
\vdots & \vdots & \ddots & \vdots \\
\left(x_{0}, y_{q-1}\right) & \left(x_{1}, g\left(x_{0}, y_{q-1}\right)\right) & \ldots & \left(x_{p-1}, g\left(x_{p-2}, \ldots g\left(x_{1}, g\left(x_{0}, y_{q-1}\right)\right) \ldots\right)\right) .
\end{array}
$$

The orbits of the one-dimensional maps $f$ and $g_{p}$ determine the orbits of the triangular map $T$, as we show in the following Lemma:

Lemma 2.1. Let $T=(f, g): X \times Y \longrightarrow X \times Y$ be a continuous triangular map. Then the following hold:

(1) If $f$ has a periodic orbit $P$ and $g_{p}$ has a periodic orbit $Q$, then P.Q is a periodic orbit of $T$.

(2) Conversely, each periodic orbit of $T$ can be obtained as a product of a periodic orbit $P$ of $f$ by a periodic orbit of $g_{p}$. 
Proof. See Alsedà-Llibre ${ }^{1}$.

The topological entropy is a measure of complexity of a dynamical system. Let $T$ be a triangular map like defined in the earlier Lemma. The Bowen's formula for the inferior and superior values of the topological entropy of $T, h_{t o p}(T)$, is valid, that is,

$$
\max \left\{h_{\text {top }}(f), h_{\text {top }}\left(g_{p}\right)\right\} \leq h_{\text {top }}(T) \leq h_{\text {top }}(f)+h_{\text {top }}\left(g_{p}\right),
$$

where $h_{t o p}(f)$ and $h_{t o p}\left(g_{p}\right)$ represent, respectively, the topological entropy of the basis map, $f$, and the topological entropy of the fiber map associated to the orbit $P, g_{p}$.

\section{Chaotic behavior in the map $F$}

It is possible to prove the existence of chaotic behavior in the map $F$ if there are parameter values that correspond to a positive value of the topological entropy.

Let us consider first the basis map $f$. It is an one-dimensional map and it can be easily proved that $f$ is a bimodal map when $\alpha \sigma-1>0$. In this case there are two turning points, whose expressions are:

$$
c_{1}=\mu-\sqrt{\frac{\alpha}{\alpha \sigma-1}-1} \text { and } c_{2}=\mu+\sqrt{\frac{\alpha}{\alpha \sigma-1}-1} .
$$

The first one corresponds to the relative minimum and the second corresponds to the relative maximum. Fixing the parameters $\mu=100$ and $\sigma=0.3, f$ depends only on $\alpha$ which is the most interesting parameter from the economics point of view. In Fig. 3 we present the long term behavior of the map $f$ when $\alpha$ changes between 7 and 14 .

For $\alpha=11.1726, f$ has an orbit, $P=\left\{y_{0}, y_{1}, y_{2}, y_{3}\right\}$, of period 4 , such that $f\left(f\left(c_{1}\right)\right)=c_{2}$, described in Fig.4. The corresponding kneading data $(A L B R, B R A L)$ generates a three interval Markov partition of the line $y$, which corresponds to the transition matrix:

$$
M(f)=\left[\begin{array}{lll}
1 & 1 & 0 \\
1 & 1 & 1 \\
0 & 1 & 1
\end{array}\right]
$$

The maximum eigenvalue is $\lambda_{y}=2.41421$ and the value of the topological entropy of the basis map is

$$
h_{t o p}(f)=\log \left(\lambda_{y}\right)=0.881374 \ldots
$$




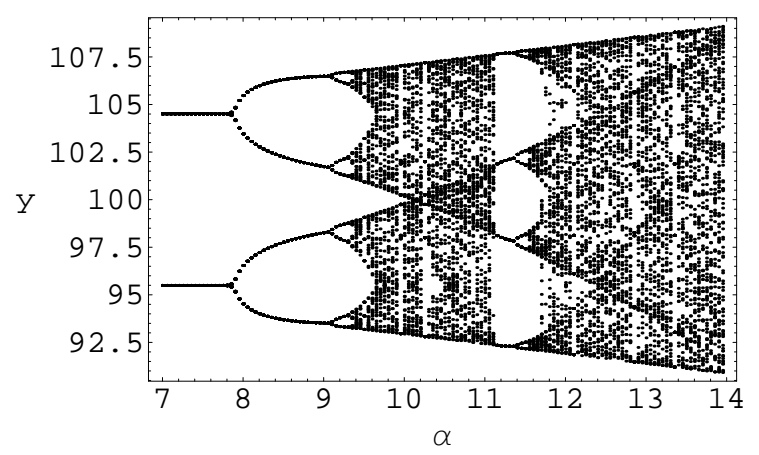

Figure 3. Bifurcation diagram of the basis map $f$ as a function of $\alpha$, with initial conditions $c_{1}$ and $c_{2}$.

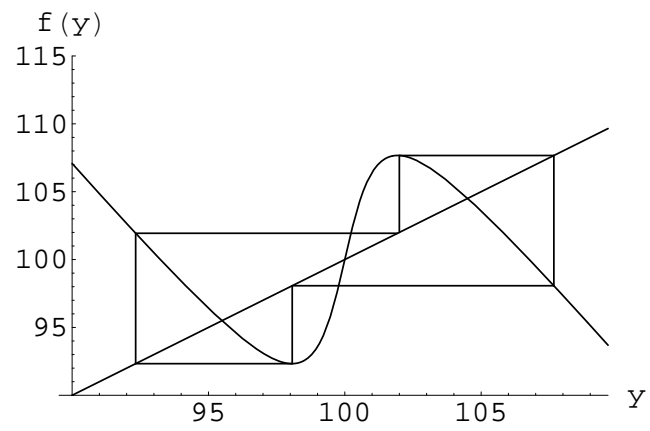

Figure 4. Period-4 orbit of the map $f$, when $\alpha=11.1726, \mu=100$ and $\sigma=0.3$.

Let us consider now the points of the orbit $P$ ordered like:

$$
y_{0}=92, \ldots ; \quad y_{1}=101, \ldots ; \quad y_{3}=107, \ldots ; \quad y_{4}=98, \ldots .
$$

Consider also the function $g_{p_{0}}(k)$, depending on the variable $k$ and defined, according to (6), by

$$
g_{p_{0}}(k)=g\left(y_{3}, g\left(y_{2}, g\left(y_{1}, g\left(y_{0}, k\right)\right)\right)\right) .
$$

If we fix $\beta=9.5$ and $\delta=0.52$, the bifurcation diagram of $g_{p_{0}}$ as a function of $\gamma$, the free parameter, is presented in Fig. 5 . The study of this function can give us significant information about the long term behavior of the original map $F$. Let's consider, for example, the case $\gamma=0.8518$. The function 


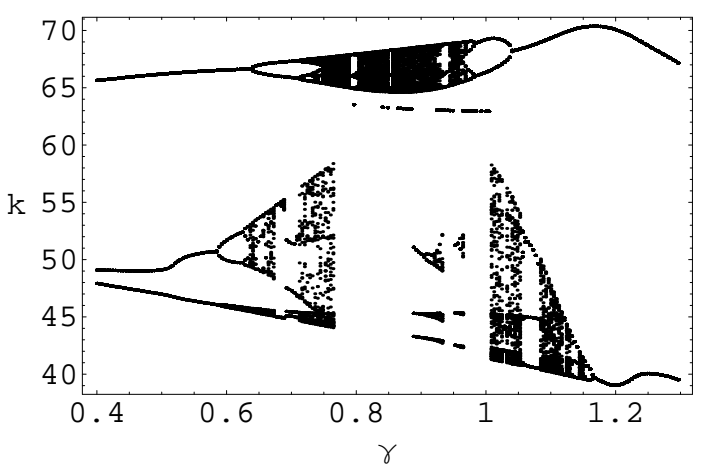

Figure 5. Bifurcation diagram of the map $g_{p_{0}}(k)$ as a function of $\gamma$.

$g_{p_{0}}(k)$, whose graphical representation is showed in Fig.6 is continuous, limited and has many critical points but its dynamics reduces to the values where there is an intersection with the diagonal line. If we iterate all the critical points of $g_{p_{0}}(k)$ it is all reduced to an orbit of period 4 or to a fix point (see Fig.7).

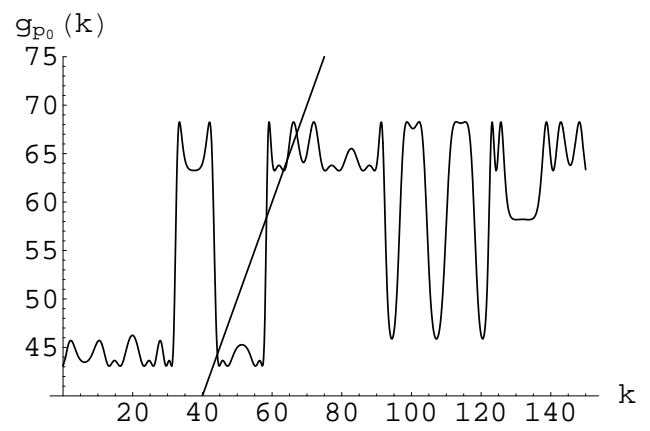

Figure 6. Graphical representation of the map $g_{p_{0}}(k)$ for $\gamma=0.8518$.

It is very interesting to notice what happens if we change the order of appearance of the points $y_{0}, y_{1}, y_{2}, y_{3}$, of the orbit $P$, in the construction of the function $g_{p}(k)$. We have three more possibilities. We can also define 


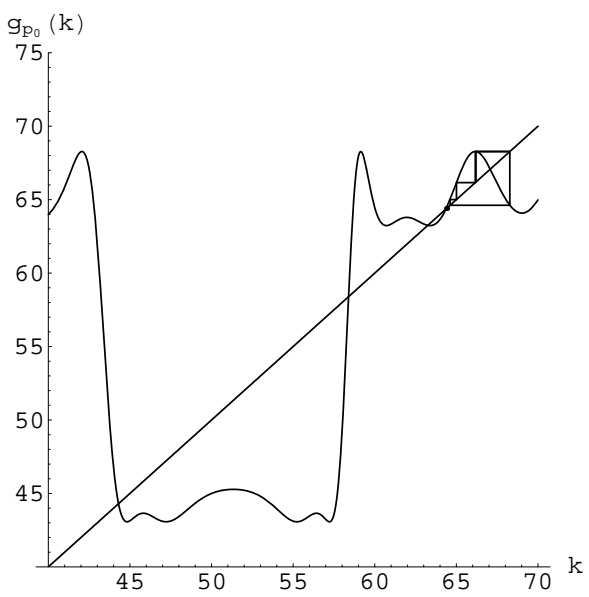

Figure 7. A magnification of the graphical representation of the map $g_{p_{0}}(k)$ for $\gamma=$ 0.8518 .

$g_{p_{1}}(k), g_{p_{2}}(k)$ and $g_{p_{3}}(k)$ in the following way:

$$
\begin{aligned}
g_{p_{1}}(k) & =g\left(y_{0}, g\left(y_{3}, g\left(y_{2}, g\left(y_{1}, k\right)\right)\right)\right), \\
g_{p 2}(k) & =g\left(y_{1}, g\left(y_{0}, g\left(y_{3}, g\left(y_{2}, k\right)\right)\right)\right), \\
\text { and } \quad g_{p 3}(k) & =g\left(y_{2}, g\left(y_{1}, g\left(y_{0}, g\left(y_{3}, k\right)\right)\right)\right) .
\end{aligned}
$$

We obtain functions, all very different from each other, but the dynamic behavior of all these maps asymptotically goes to a period 4 orbit or to a fix point. In Fig.10 are shown the stable orbits for the considered parameter values. The periods of the orbits are in agreement with the considerations made in Section 2: the period of the orbit of $F$ is equal to 16 or to 4 depending on the initial conditions. In each fiber we can see the orbit of period 4 , in black, and the fix point, in grey. The first fiber corresponds to the stable orbits of the function $g_{p_{0}}(k)$ and the others respectively to the stable orbits of functions $g_{p_{3}}(k), g_{p_{1}}(k)$ and $g_{p_{2}}(k)$.

The transition matrix is:

$$
M(f)=\left[\begin{array}{lll}
0 & 1 & 0 \\
0 & 0 & 1 \\
1 & 1 & 1
\end{array}\right]
$$

which corresponds to a topological entropy given by:

$$
h_{t o p}\left(g_{p_{0}}\right)=\log (1.83929 \ldots)=0.609378 \ldots .
$$



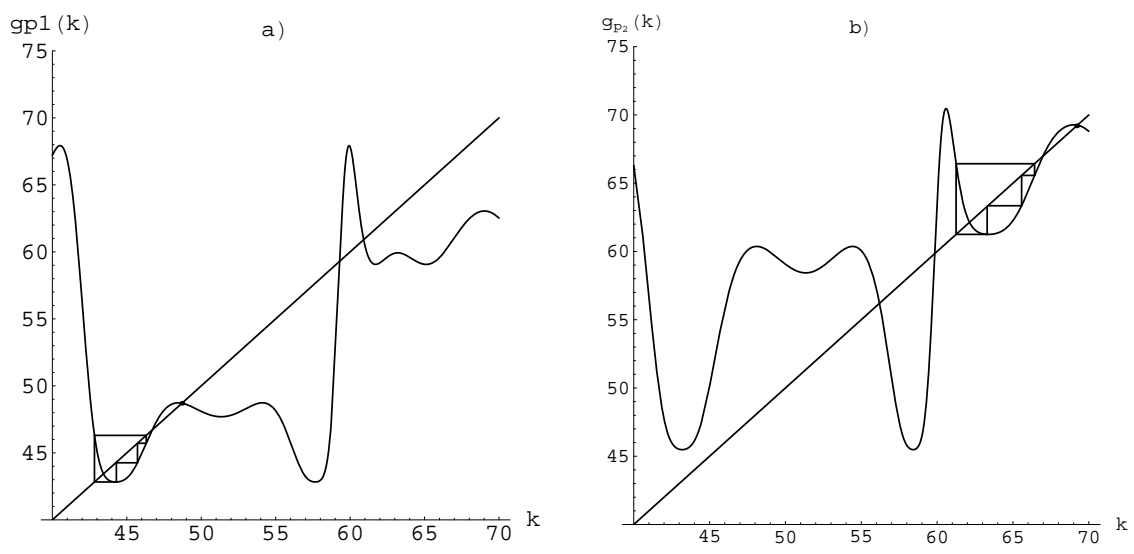

Figure 8. Graphical representation of the functions $g_{p_{1}}$ and $g_{p_{2}}$.
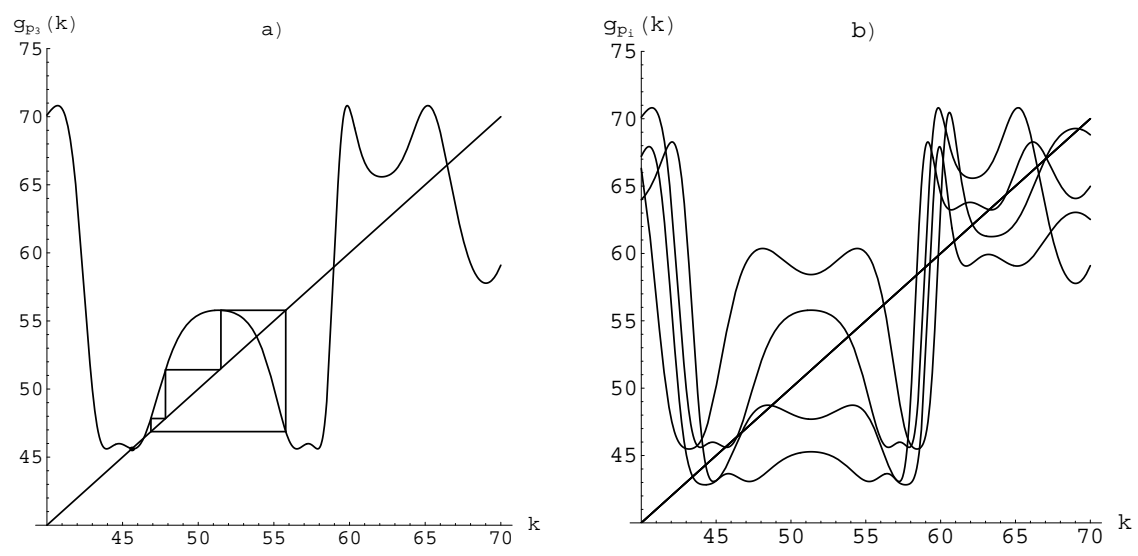

Figure 9. In plot $a$ ) graphical representation of the function $g_{p_{3}}$ and in plot $b$ ) graphical representation of the functions $g_{p_{i}}, i=0,1,2,3$ in the same plot.

The earlier value doesn't depend on the function $g_{p_{i}}$, that is, doesn't depend on the fiber.

So we can conclude that the topological entropy of the original triangular map $F$ is such that:

$$
0.881374 \ldots \leq h_{t o p}(F) \leq 1.574521 \ldots
$$




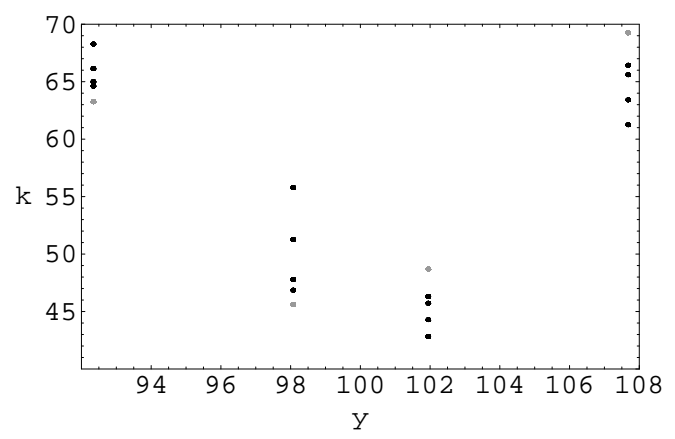

Figure 10. The stable orbits of the triangular map $F$ for $\alpha=11.1726, \mu=100, \sigma=0.3$, $\beta=9.5, \delta=0.52$ and $\gamma=0.8515$.

The map $F$ certainly has a value of the topological entropy that is positive, and therefore, the system is chaotic for these parameter values.

\section{Final conclusions}

In this paper we presented a Kaldor-type business cycle model described by a two-dimensional map. The model is a modification of the one proposed in Bischi et al..$^{2}$ and Dieci et al. ${ }^{3}$ and can be reduced to a map of triangular type with income and capital stock as variables. Due to its triangular structure it is possible to compute the topological entropy, for certain values of the parameters, as a measure of complexity of the system. The introduction of nonlinearity in the capital stock variable, into the investment and savings functions allows us to have chaotic basis map and chaotic fiber map, since we found a positive value for the topological entropy in both cases.

\section{References}

1. L. Alsedà; J. Llibre; Periods for triangular maps, Bull. Austral. Math. Soc. (1993), no. 47, 41-53.

2. GI. Bischi; R. Dieci; R. Rodano; S. Saltari; Multiple attractors and global bifurcations in a Kaldor-type business cycle model, J. Evol Econ (2001), no. $11,527-554$.

3. R. Dieci; GI. Bischi; L. Gardini; From bi-stability to chaotic oscillations in a macroeconomic model, Chaos Solitons Fractals 21 (2004), no. 2, 403-412.

4. D. Mendes; J. Sousa Ramos; Kneading theory for triangular maps, Int. J. Pure Appl. Math. 10 (2) (2004) 421-450.

5. N. Kaldor; The model of the trade cycle, Economic Journal, 50 (1940), 78-92. 
6. J. P. Lampreia; J. Sousa Ramos; Symbolic dynamics of bimodal maps, Portugal. Math. 54 (1997), no. 1, 1-18.

7. C. Mira; L. Gardini; A. Barugola; J.-C. Cathala; Chaotic dynamics in twodimensional noninvertible maps. World Scientific Series on Nonlinear Science. Series A: Monographs and Treatises, 20. World Scientific Publishing Co., Inc., River Edge, NJ, 1996. xiv+607 pp.

8. J. Milnor; W. Thurston; On iterated maps of the interval. Dynamical systems (College Park, MD, 1986-87), 465-563, Lecture Notes in Math., 1342, Springer, Berlin, 1988.

9. T. Puu; Attractors, bifurcations, and chaos. Nonlinear phenomena in economics. Springer-Verlag, Berlin, 2000. xii+507 pp. 